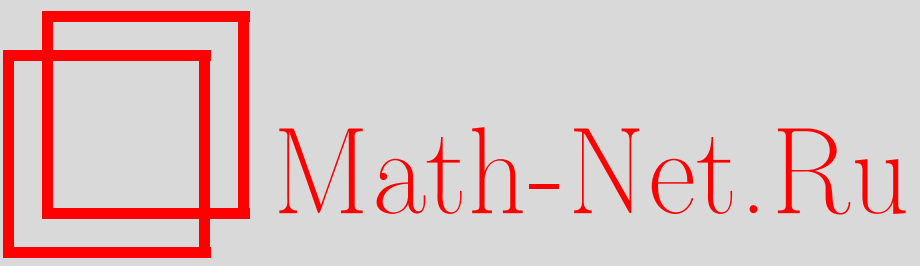

О. И. Мохов, Согласованные нелокальные скобки Пуассона гидродинамического типа и связанные с ними интегрируемые иерархии, ТМФ, 2002, том 132, номер 1, 60-73

DOI: https://doi.org/10.4213/tmf346

Использование Общероссийского математического портала Math-Net.Ru подразумевает, что вы прочитали и согласны с пользовательским соглашением

http: //www . mathnet.ru/rus/agreement

Параметры загрузки:

IP: 3.91 .87 .62

26 апреля 2023 г., 10:22:26 


\section{СОГЛАСОВАННЫЕ НЕЛОКАЛЬНЫЕ СКОБКИ ПУАССОНА ГИДРОДИНАМИЧЕСКОГО ТИПА И СВЯЗАННЫЕ С НИМИ ИНТЕГРИРУЕМЫЕ ИЕРАРХИИ}

Построены интегрируемые бигамильтоновы иерархии, связанные с согласованными нелокальными скобками Пуассона гидродинамического типа. Решена задача о канонической форме для пары согласованных нелокальных скобок Пуассона гидродинамического типа. Получена система уравнений, описывающая согласованные нелокальные скобки Пуассона гидродинамического типа и интегрируемая методом обратной задачи рассеяния. Любое решение этой интегрируемой системы порождает по явным формулам интегрируемые бигамильтоновы системы гидродинамического типа. Построена теория скобок Пуассона специального лиувиллева вида, играющая важную роль в построении интегрируемых иерархий.

Ключевые слова: согласованные скобки Пуассона, системы гидродинамического типа, согласованные метрики, интегрируемые иерархии, бигамильтоновы структуры, нелокальные скобки Пуассона гидродинамического типа.

\section{1. ВВЕДЕНИЕ. ОСНОВНЫЕ ОПРЕДЕЛЕНИЯ}

В настоящей работе эффективно построены интегрируемые бигамильтоновы иерархии, связанные с согласованными нелокальными скобками Пуассона гидродинамического типа. Для этого решается задача о канонической форме специального вида для согласованных нелокальных скобок Пуассона гидродинамического типа. Согласованные пары скобок Пуассона гидродинамического типа проще описываются в специальных координатах, в которых соответствуюшие этим скобкам метрики диагональны (см. [1], [2]), но для эффективного построения иерархий необходим другой подход, развиваемый в данной работе. Для согласованных локальных скобок Пуассона гидродинамического типа (скобок Дубровина-Новикова [3]) соответствуюшие интегрируемые бигамильтоновы иерархии построены автором в работах [4], [5], а для согласованных нелокальных скобок Мохова-Ферапонтова [6], порождаемых метриками постоянной римановой кривизны, бигамильтоновы иерархии построены в работе [7].

${ }^{*}$ Институт теоретической физики им. Л. Д. Ландау РАН, Москва, Россия. E-mail: mokhov@mi.ras.ru, mokhov@landau.ac.ru 
1.1. Локальные скобки Пуассона гидродинамического типа. Локальной скобкой Пуассона гидродинамического типа или скобкой Дубровина-Новикова [3] называется произвольная локальная однородная скобка Пуассона первого порядка, т.е. скобка Пуассона вида

$$
\left\{u^{i}(x), u^{j}(y)\right\}=g^{i j}(u(x)) \delta_{x}(x-y)+b_{k}^{i j}(u(x)) u_{x}^{k} \delta(x-y),
$$

где $u^{1}, \ldots, u^{N}$ - локальные координаты на некотором заданном гладком $N$-мерном многообразии $M ; u^{i}(x), \quad 1 \leqslant i \leqslant N,-$ функции (поля) одной независимой переменной $x$; коэфрициенты $g^{i j}(u)$ и $b_{k}^{i j}(u)$ - гладкие функции локальных координат.

Другими словами, для произвольных функционалов $I[u]$ и $J[u]$ на пространстве полей $u^{i}(x), 1 \leqslant i \leqslant N$, определяется скобка вида

$$
\{I, J\}=\int \frac{\delta I}{\delta u^{i}(x)}\left(g^{i j}(u(x)) \frac{d}{d x}+b_{k}^{i j}(u(x)) u_{x}^{k}\right) \frac{\delta J}{\delta u^{j}(x)} d x
$$

и требуется, чтобы она являлась скобкой Пуассона, т.е. была кососимметричной:

$$
\{I, J\}=-\{J, I\}
$$

и удовлетворяла тождеству Якоби

$$
\{\{I, J\}, K\}+\{\{J, K\}, I\}+\{\{K, I\}, J\}=0
$$

для произвольных функционалов $I[u], J[u]$ и $K[u]$.

Локальная скобка (1.2) называется невырожденной, если $\operatorname{det}\left(g^{i j}(u)\right) \not \equiv 0$. Для обших невырожденных скобок (1.2) Дубровин и Новиков доказали следуюшую важную теорему.

Теорема 1.1 [3]. Если $\operatorname{det}\left(g^{i j}(u)\right) \not \equiv 0$, то скобка (1.2) является скобкой Пуассона, т.е. она кососимметрична и удовлетворяет тождеству Якоби, тогда и только тогда, когда

1) $g^{i j}(u)$ - произвольная плоская псевдориманова контравариантная метрика (метрика нулевой римановой кривизнь);

2) $b_{k}^{i j}(u)=-g^{i s}(u) \Gamma_{s k}^{j}(u)$, әде $\Gamma_{s k}^{j}(u)-$ риманова связность, порожсдаемая контравариантной метрикой $g^{i j}(u)$ (связность Леви-Чивита).

Следовательно, для любой невырожденной локальной скобки Пуассона гидродинамического типа всегда сушествуют локальные координаты $v^{1}, \ldots, v^{N}$ (плоские координаты метрики $\left.g^{i j}(u)\right)$, в которых коэффициенты скобки постоянны:

$$
\widetilde{g}^{i j}(v)=\eta^{i j}=\mathrm{const}, \quad \widetilde{\Gamma}_{j k}^{i}(v)=0, \quad \tilde{b}_{k}^{i j}(v)=0
$$

т.е. скобка имеет вид

$$
\{I, J\}=\int \frac{\delta I}{\delta v^{i}(x)} \eta^{i j} \frac{d}{d x} \frac{\delta J}{\delta v^{j}(x)} d x
$$


где $\eta^{i j}$ - невырожденная симметричная постоянная матрица:

$$
\eta^{i j}=\eta^{j i}, \quad \eta^{i j}=\text { const }, \quad \operatorname{det}\left(\eta^{i j}\right) \neq 0 .
$$

Локальные скобки Пуассона гидродинамического типа (1.1) введены и изучены Дубровиным и Новиковым в работе [3], где предложен соответствующий структурам (1.1) общий локальный гамильтонов подход к так называемым однороднымм системам гидродинамического типа, т.е. эволюционным квазилинейным системам уравнений в частных производных первого порядка

$$
u_{t}^{i}=V_{j}^{i}(u) u_{x}^{j}
$$

Этот гамильтонов подход был мотивирован изучением уравнений эйлеровой гидродинамики и уравнений усреднения Уизема, описывающих эволюцию медленно промодулированных многофазных решений уравнений в частных производных (см. [8]). В работах [9], [10] построена теория интегрирования диагонализуемых гамильтоновых (а также полугамильтоновых) однородных систем гидродинамического типа.

1.2. Нелокальные скобки Пуассона гидродинамического типа. Нелокальные скобки Пуассона гидродинамического типа (скобки Мохова-Ферапонтова) были введены и изучены в работе [6] (см. также [11]-[13]). Они имеют следующий вид:

$$
\{I, J\}=\int \frac{\delta I}{\delta u^{i}(x)}\left(g^{i j}(u(x)) \frac{d}{d x}+b_{k}^{i j}(u(x)) u_{x}^{k}+K u_{x}^{i}\left(\frac{d}{d x}\right)^{-1} u_{x}^{j}\right) \frac{\delta J}{\delta u^{j}(x)} d x
$$

где $K$ - произвольная константа.

Скобка вида (1.7) называется невырожденной, если $\operatorname{det}\left(g^{i j}(u)\right) \not \equiv 0$.

Tеорема 1.2 [6]. Если $\operatorname{det}\left(g^{i j}(u)\right) \not \equiv 0$, то скобка (1.7) является скобкой Пуассона, т.е. она кососимметрична и удовлетворяет тождеству Якоби, тогда и только тогда, когда

1) $g^{i j}(u)$ - произвольная псевдориманова контравариантная метрика постоянной римановой кривизны $K$;

2) $b_{k}^{i j}(u)=-g^{i s}(u) \Gamma_{s k}^{j}(u)$, әде $\Gamma_{s k}^{j}(u)-$ риманова связность, порожсдаемая контравариантной метрикой $g^{i j}(u)$ (связность Леви-Чивита).

Ферапонтов [11] ввел и изучил более общие нелокальные скобки Пуассона гидродинамического типа (скобки Ферапонтова), а именно скобки Пуассона вида

$$
\begin{aligned}
&\{I, J\}= \int \frac{\delta I}{\delta u^{i}(x)}\left(g^{i j}(u(x)) \frac{d}{d x}+b_{k}^{i j}(u(x)) u_{x}^{k}+\right. \\
&\left.+\sum_{\alpha=1}^{L} \varepsilon_{\alpha}\left(w^{\alpha}\right)_{k}^{i}(u(x)) u_{x}^{k}\left(\frac{d}{d x}\right)^{-1}\left(w^{\alpha}\right)_{s}^{j}(u(x)) u_{x}^{s}\right) \frac{\delta J}{\delta u^{j}(x)} d x, \\
& \operatorname{det}\left(g^{i j}(u)\right) \not \equiv 0,
\end{aligned}
$$

где $\varepsilon_{\alpha}= \pm 1,1 \leqslant \alpha \leqslant L$. 
Теорема 1.3 [11]. СКобка (1.8) является скобкой Пуассона, т.е. она кососимметрична и удовлетворяет тождеству Якоби, тогда и только тогда, когда

1) $b_{k}^{i j}(u)=-g^{i s}(u) \Gamma_{s k}^{j}(u)$, әде $\Gamma_{s k}^{j}(u)-$ риманова связность, порождаемая контравариантной метрикой $g^{i j}(u)$ (связность Леви-Чивита);

2) метрика $g^{i j}(u)$ и набор аффиноров $\left(w^{\alpha}\right)_{j}^{i}(u)$ удовлетворяют соотношениям

$$
\begin{gathered}
g_{i k}(u)\left(w^{\alpha}\right)_{j}^{k}(u)=g_{j k}(u)\left(w^{\alpha}\right)_{i}^{k}(u), \quad \alpha=1, \ldots, L, \\
\nabla_{k}\left(w^{\alpha}\right)_{j}^{i}(u)=\nabla_{j}\left(w^{\alpha}\right)_{k}^{i}(u), \quad \alpha=1, \ldots, L, \\
R_{k l}^{i j}(u)=\sum_{\alpha=1}^{L} \varepsilon_{\alpha}\left(\left(w^{\alpha}\right)_{l}^{i}(u)\left(w^{\alpha}\right)_{k}^{j}(u)-\left(w^{\alpha}\right)_{l}^{j}(u)\left(w^{\alpha}\right)_{k}^{i}(u)\right) .
\end{gathered}
$$

Кроме того, семейство аффиноров $w^{\alpha}(u)$ коммутативное: $\left[w^{\alpha}, w^{\beta}\right]=0$.

Выпишем все соотношения на коэффициенты нелокальной скобки Пуассона (1.8) в удобном для дальнейшего использования виде.

Лемма 1.1. Скобка вида (1.8) является скобкой Пуассона тогда и только тогда, когда ее коэффичиенты удовлетворяют соотношениям

$$
\begin{gathered}
g^{i j}=g^{j i} \\
\frac{\partial g^{i j}}{\partial u^{k}}=b_{k}^{i j}+b_{k}^{j i}, \\
g^{i s} b_{s}^{j k}=g^{j s} b_{s}^{i k} \\
g^{i s}\left(w^{\alpha}\right)_{s}^{j}=g^{j s}\left(w^{\alpha}\right)_{s}^{i}, \\
\left(w^{\alpha}\right)_{s}^{i}\left(w^{\beta}\right)_{j}^{s}=\left(w^{\beta}\right)_{s}^{i}\left(w^{\alpha}\right)_{j}^{s}, \\
g^{i s} g^{j r} \frac{\partial\left(w^{\alpha}\right)_{r}^{k}}{\partial u^{s}}-g^{j r} b_{s}^{i k}\left(w^{\alpha}\right)_{r}^{s}=g^{j s} g^{i r} \frac{\partial\left(w^{\alpha}\right)_{r}^{k}}{\partial u^{s}}-g^{i r} b_{s}^{j k}\left(w^{\alpha}\right)_{r}^{s}, \\
g^{i s}\left(\frac{\partial b_{r}^{j k}}{\partial u^{s}}-\frac{\partial b_{s}^{j k}}{\partial u^{r}}\right)+b_{s}^{i k} b_{r}^{s j}-b_{s}^{i j} b_{r}^{s k}=\sum_{\alpha=1}^{L} \varepsilon_{\alpha} g^{i s}\left(\left(w^{\alpha}\right)_{s}^{j}\left(w^{\alpha}\right)_{r}^{k}-\left(w^{\alpha}\right)_{r}^{j}\left(w^{\alpha}\right)_{s}^{k}\right) .
\end{gathered}
$$

1.3. Согласованные скобки Пуассона. Магри предложил [14] бигамильтонов подход к интегрированию нелинейных систем, показав, что интегрируемость тесно связана с бигамильтоновостью, т.е. наличием у системы двух согласованных гамильтоновых представлений.

ОПРЕДЕЛЕнИЕ [14]. Две скобки Пуассона $\{\cdot, \cdot\}_{1}$ и $\{\cdot, \cdot\}_{2}$ называются согласованными, если произвольная линейная комбинация этих скобок Пуассона

$$
\{\cdot, \cdot\}=\lambda_{1}\{\cdot, \cdot\}_{1}+\lambda_{2}\{\cdot, \cdot\}_{2},
$$

где $\lambda_{1}$ и $\lambda_{2}-$ произвольные константы, также всегда является скобкой Пуассона. В этом случае говорят также, что скобки $\{\cdot, \cdot\}_{1}$ и $\{\cdot, \cdot\}_{2}$ образуют пучок скобок Пуассона. 
Как показал Магри, согласованные скобки Пуассона порождают интегрируемые иерархии систем дифференциальных уравнений. В частности, бигамильтоновость системы порождает рекуррентные соотношения для законов сохранения этой системы. В данной работе построены интегрируемые иерархии, отвечающие согласованным нелокальным скобкам Пуассона гидродинамического типа.

\section{2. ПУЧОК НЕЛОКАЛЬНЫХ СКОБОК ПУАССОНА ГИДРОДИНАМИЧЕСКОГО ТИПА}

Опишем все нелокальные скобки Пуассона (1.8), согласованные с постоянной невырожденной скобкой Пуассона гидродинамического типа

$$
\{I, J\}_{2}=\int \frac{\delta I}{\delta u^{i}(x)} \eta^{i j} \frac{d}{d x} \frac{\delta J}{\delta u^{j}(x)} d x
$$

где $\eta^{i j}$ - произвольная невырожденная симметричная постоянная матрища (1.6), т.е. классифицируем все пучки нелокальных скобок Пуассона

$$
\{I, J\}_{\lambda}=\{I, J\}_{1}+\lambda\{I, J\}_{2}
$$

где $\{I, J\}_{1}$ - скобка Пуассона вида (1.8).

ЛЕмма 2.1. Скобки Пуассона (2.1) и (1.8) согласованы тогда и только тогда, когда выполнены следующие соотношения:

$$
\begin{gathered}
\eta^{i s} b_{s}^{j k}=\eta^{j s} b_{s}^{i k}, \\
\eta^{i s}\left(w^{\alpha}\right)_{s}^{j}=\eta^{j s}\left(w^{\alpha}\right)_{s}^{i}, \\
\frac{\partial\left(w^{\alpha}\right)_{j}^{i}}{\partial u^{k}}=\frac{\partial\left(w^{\alpha}\right)_{k}^{i}}{\partial u^{j}}, \\
\frac{\partial b_{r}^{j k}}{\partial u^{s}}-\frac{\partial b_{s}^{j k}}{\partial u^{r}}=\sum_{\alpha=1}^{L} \varepsilon_{\alpha}\left(\left(w^{\alpha}\right)_{s}^{j}\left(w^{\alpha}\right)_{r}^{k}-\left(w^{\alpha}\right)_{r}^{j}\left(w^{\alpha}\right)_{s}^{k}\right) .
\end{gathered}
$$

Важно отметить, что соотношение

$$
\eta^{j r} b_{s}^{i k}\left(w^{\alpha}\right)_{r}^{s}=\eta^{i r} b_{s}^{j k}\left(w^{\alpha}\right)_{r}^{s}
$$

получаемое из (1.17) как одно из условий согласованности скобок Пуассона (2.1) и (1.8), следует из формул (2.3)-(2.5) для любых скобок Пуассона (1.8). Действительно, из (2.4) получаем

$$
\eta^{j r} b_{s}^{i k}\left(w^{\alpha}\right)_{r}^{s}=\eta^{s r} b_{s}^{i k}\left(w^{\alpha}\right)_{r}^{j}
$$

а из (2.3) имеем

$$
\eta^{s r} b_{s}^{i k}\left(w^{\alpha}\right)_{r}^{j}=\eta^{s i} b_{s}^{r k}\left(w^{\alpha}\right)_{r}^{j}
$$

Следовательно, соотношение (2.7) сводится к равенству

$$
\eta^{s i} b_{s}^{r k}\left(w^{\alpha}\right)_{r}^{j}=\eta^{i r} b_{s}^{j k}\left(w^{\alpha}\right)_{r}^{s}
$$


т.е.

$$
b_{s}^{r k}\left(w^{\alpha}\right)_{r}^{j}=b_{r}^{j k}\left(w^{\alpha}\right)_{s}^{r} .
$$

Докажем, что соотношение (2.11) выполняется для любой скобки Пуассона (1.8), для которой имеет место равенство (2.5). Действительно, в этом случае соотношение (1.17) для скобки Пуассона (1.8) принимает вид

$$
g^{j r} b_{s}^{i k}\left(w^{\alpha}\right)_{r}^{s}=g^{i r} b_{s}^{j k}\left(w^{\alpha}\right)_{r}^{s}
$$

Из (1.15) получаем

$$
g^{j r} b_{s}^{i k}\left(w^{\alpha}\right)_{r}^{s}=g^{s r} b_{s}^{i k}\left(w^{\alpha}\right)_{r}^{j}
$$

а из (1.14) имеем

$$
g^{s r} b_{s}^{i k}\left(w^{\alpha}\right)_{r}^{j}=g^{s i} b_{s}^{r k}\left(w^{\alpha}\right)_{r}^{j},
$$

т.е. соотношение (2.12) сведено к выражению

$$
g^{s i} b_{s}^{r k}\left(w^{\alpha}\right)_{r}^{j}=g^{i r} b_{s}^{j k}\left(w^{\alpha}\right)_{r}^{s},
$$

которое эквивалентно формуле (2.11).

\section{3. КАНОНИЧЕСКАЯ ФОРМА СОГЛАСОВАННЫХ ПАР СКОБОК}

Теорема 3.1. Произвольная нелокальная скобка Пуассона $\{I, J\}_{1}$ вида (1.8) согласована с постоянной скобкой Пуассона (2.1) тогда и только тогда, когда она имеет вид

$$
\begin{aligned}
\{I, J\}_{1}=\int & \frac{\delta I}{\delta u^{i}(x)}\left(\left[\eta^{i s} \frac{\partial F^{j}}{\partial u^{s}}+\eta^{j s} \frac{\partial F^{i}}{\partial u^{s}}-\eta^{i s} \eta^{j k} \sum_{\alpha=1}^{L} \varepsilon_{\alpha} \frac{\partial \psi^{\alpha}}{\partial u^{s}} \frac{\partial \psi^{\alpha}}{\partial u^{k}}\right] \frac{d}{d x}+\right. \\
& +\left[\eta^{i s} \frac{\partial^{2} F^{j}}{\partial u^{s} \partial u^{k}}-\eta^{i s} \eta^{j p} \sum_{\alpha=1}^{L} \varepsilon_{\alpha} \frac{\partial^{2} \psi^{\alpha}}{\partial u^{s} \partial u^{k}} \frac{\partial \psi^{\alpha}}{\partial u^{p}}\right] u_{x}^{k}+ \\
& \left.+\eta^{i p} \eta^{j r} \sum_{\alpha=1}^{L} \varepsilon_{\alpha} \frac{\partial^{2} \psi^{\alpha}}{\partial u^{p} \partial u^{k}} u_{x}^{k}\left(\frac{d}{d x}\right)^{-1} \frac{\partial^{2} \psi^{\alpha}}{\partial u^{r} \partial u^{s}} u_{x}^{s}\right) \frac{\delta J}{\delta u^{j}(x)} d x
\end{aligned}
$$

где $F^{i}(u), \quad 1 \leqslant i \leqslant N$, и $\psi^{\alpha}(u), \quad 1 \leqslant \alpha \leqslant L,-$ гладкие функиии, определеннъе в некоторой области локальных координат.

Из соотношения (2.5) немедленно следует, что локально сушествуют функции $\left(\varphi^{\alpha}\right)^{i}(u), 1 \leqslant i \leqslant N, 1 \leqslant \alpha \leqslant L$, такие, что

$$
\left(w^{\alpha}\right)_{j}^{i}=\frac{\partial\left(\varphi^{\alpha}\right)^{i}}{\partial u^{j}}
$$

Тогда соотношение (2.6) принимает вид

$$
\frac{\partial b_{r}^{j k}}{\partial u^{s}}-\frac{\partial b_{s}^{j k}}{\partial u^{r}}=\sum_{\alpha=1}^{L} \varepsilon_{\alpha}\left(\frac{\partial\left(\varphi^{\alpha}\right)^{j}}{\partial u^{s}} \frac{\partial\left(\varphi^{\alpha}\right)^{k}}{\partial u^{r}}-\frac{\partial\left(\varphi^{\alpha}\right)^{j}}{\partial u^{r}} \frac{\partial\left(\varphi^{\alpha}\right)^{k}}{\partial u^{s}}\right) .
$$

3 Теоретическая и математическая физика, т. 132, № 1, 2002 г. 
Введем функцию

$$
A_{k}^{i j}(u)=b_{k}^{i j}(u)-\sum_{\alpha=1}^{L} \varepsilon_{\alpha}\left(\varphi^{\alpha}\right)^{i} \frac{\partial\left(\varphi^{\alpha}\right)^{j}}{\partial u^{k}} .
$$

Тогда, используя (3.3), получаем

$$
\begin{aligned}
\frac{\partial A_{k}^{i j}}{\partial u^{l}} & =\frac{\partial b_{k}^{i j}}{\partial u^{l}}-\sum_{\alpha=1}^{L} \varepsilon_{\alpha} \frac{\partial\left(\varphi^{\alpha}\right)^{i}}{\partial u^{l}} \frac{\partial\left(\varphi^{\alpha}\right)^{j}}{\partial u^{k}}-\sum_{\alpha=1}^{L} \varepsilon_{\alpha}\left(\varphi^{\alpha}\right)^{i} \frac{\partial^{2}\left(\varphi^{\alpha}\right)^{j}}{\partial u^{k} \partial u^{l}}= \\
& =\frac{\partial b_{l}^{i j}}{\partial u^{k}}-\sum_{\alpha=1}^{L} \varepsilon_{\alpha} \frac{\partial\left(\varphi^{\alpha}\right)^{i}}{\partial u^{k}} \frac{\partial\left(\varphi^{\alpha}\right)^{j}}{\partial u^{l}}-\sum_{\alpha=1}^{L} \varepsilon_{\alpha}\left(\varphi^{\alpha}\right)^{i} \frac{\partial^{2}\left(\varphi^{\alpha}\right)^{j}}{\partial u^{k} \partial u^{l}}=\frac{\partial A_{l}^{i j}}{\partial u^{k}}
\end{aligned}
$$

Следовательно, существуют функции $P^{i j}(u), 1 \leqslant i, j \leqslant N$, такие, что

$$
A_{k}^{i j}(u)=\frac{\partial P^{i j}}{\partial u^{k}}
$$

Таким образом,

$$
b_{k}^{i j}(u)=\frac{\partial P^{i j}}{\partial u^{k}}+\sum_{\alpha=1}^{L} \varepsilon_{\alpha}\left(\varphi^{\alpha}\right)^{i} \frac{\partial\left(\varphi^{\alpha}\right)^{j}}{\partial u^{k}} .
$$

Из соотношения (1.13) для скобки Пуассона $\{I, J\}_{1}$ получаем

$$
\frac{\partial g^{i j}}{\partial u^{k}}=b_{k}^{i j}+b_{k}^{j i}=\frac{\partial P^{i j}}{\partial u^{k}}+\frac{\partial P^{j i}}{\partial u^{k}}+\sum_{\alpha=1}^{L} \varepsilon_{\alpha}\left(\varphi^{\alpha}\right)^{i} \frac{\partial\left(\varphi^{\alpha}\right)^{j}}{\partial u^{k}}+\sum_{\alpha=1}^{L} \varepsilon_{\alpha}\left(\varphi^{\alpha}\right)^{j} \frac{\partial\left(\varphi^{\alpha}\right)^{i}}{\partial u^{k}}
$$

т.е., используя (1.12), имеем

$$
g^{i j}=P^{i j}+P^{j i}+\sum_{\alpha=1}^{L} \varepsilon_{\alpha}\left(\varphi^{\alpha}\right)^{i}\left(\varphi^{\alpha}\right)^{j}+c^{i j}+c^{j i},
$$

где $c^{i j}$-произвольная постоянная матрица, $c^{i j}=$ const. Определим функцию $R^{i j}(u)$ :

$$
R^{i j}=P^{i j}+c^{i j}
$$

и получим доказательство лиувиллевости скобки Пуассона $\{I, J\}_{1}$ в рассматриваемых локальных координатах:

$$
\begin{gathered}
g^{i j}=R^{i j}+R^{j i}+\sum_{\alpha=1}^{L} \varepsilon_{\alpha}\left(\varphi^{\alpha}\right)^{i}\left(\varphi^{\alpha}\right)^{j} \\
b_{k}^{i j}=\frac{\partial R^{i j}}{\partial u^{k}}+\sum_{\alpha=1}^{L} \varepsilon_{\alpha}\left(\varphi^{\alpha}\right)^{i} \frac{\partial\left(\varphi^{\alpha}\right)^{j}}{\partial u^{k}}
\end{gathered}
$$

(подробнее о лиувиллевости см. раздел 5). 
Из соотношения (2.4) следует

$$
\eta^{i s} \frac{\partial\left(\varphi^{\alpha}\right)^{j}}{\partial u^{s}}=\eta^{j s} \frac{\partial\left(\varphi^{\alpha}\right)^{i}}{\partial u^{s}}
$$

т.e.

$$
\frac{\partial\left(\eta_{r j}\left(\varphi^{\alpha}\right)^{j}\right)}{\partial u^{p}}=\frac{\partial\left(\eta_{p j}\left(\varphi^{\alpha}\right)^{j}\right)}{\partial u^{r}}
$$

Таким образом, сушествуют функции $\psi^{\alpha}(u), 1 \leqslant \alpha \leqslant L$, такие, что

$$
\eta_{r s}\left(\varphi^{\alpha}\right)^{s}=\frac{\partial \psi^{\alpha}}{\partial u^{r}}
$$

или

$$
\left(\varphi^{\alpha}\right)^{s}=\eta^{s r} \frac{\partial \psi^{\alpha}}{\partial u^{r}}
$$

Из соотношения (2.3) следует

$$
\begin{aligned}
& \eta^{i s}\left(\frac{\partial R^{j k}}{\partial u^{s}}+\eta^{j r} \eta^{k p} \sum_{\alpha=1}^{L} \varepsilon_{\alpha} \frac{\partial \psi^{\alpha}}{\partial u^{r}} \frac{\partial^{2} \psi^{\alpha}}{\partial u^{p} \partial u^{s}}\right)= \\
& =\eta^{j s}\left(\frac{\partial R^{i k}}{\partial u^{s}}+\eta^{i r} \eta^{k p} \sum_{\alpha=1}^{L} \varepsilon_{\alpha} \frac{\partial \psi^{\alpha}}{\partial u^{r}} \frac{\partial^{2} \psi^{\alpha}}{\partial u^{p} \partial u^{s}}\right)
\end{aligned}
$$

или

$$
\frac{\partial\left(\eta_{l j} R^{j k}\right)}{\partial u^{s}}+\eta^{k p} \sum_{\alpha=1}^{L} \varepsilon_{\alpha} \frac{\partial \psi^{\alpha}}{\partial u^{l}} \frac{\partial^{2} \psi^{\alpha}}{\partial u^{p} \partial u^{s}}=\frac{\partial\left(\eta_{s j} R^{j k}\right)}{\partial u^{l}}+\eta^{k p} \sum_{\alpha=1}^{L} \varepsilon_{\alpha} \frac{\partial \psi^{\alpha}}{\partial u^{s}} \frac{\partial^{2} \psi^{\alpha}}{\partial u^{p} \partial u^{l}}
$$

Таким образом,

$$
\frac{\partial\left(\eta_{l j} R^{j k}+\eta^{k p} \sum_{\alpha=1}^{L} \varepsilon_{\alpha} \frac{\partial \psi^{\alpha}}{\partial u^{l}} \frac{\partial \psi^{\alpha}}{\partial u^{p}}\right)}{\partial u^{s}}=\frac{\partial\left(\eta_{s j} R^{j k}+\eta^{k p} \sum_{\alpha=1}^{L} \varepsilon_{\alpha} \frac{\partial \psi^{\alpha}}{\partial u^{s}} \frac{\partial \psi^{\alpha}}{\partial u^{p}}\right)}{\partial u^{l}} .
$$

Следовательно, существуют функции $F^{j}(u)$ такие, что

$$
\eta_{l j} R^{j k}+\eta^{k p} \sum_{\alpha=1}^{L} \varepsilon_{\alpha} \frac{\partial \psi^{\alpha}}{\partial u^{l}} \frac{\partial \psi^{\alpha}}{\partial u^{p}}=\frac{\partial F^{k}}{\partial u^{l}}
$$

т.е. мы получаем

$$
\begin{gathered}
R^{i j}=\eta^{i s} \frac{\partial F^{j}}{\partial u^{s}}-\eta^{i s} \eta^{j k} \sum_{\alpha=1}^{L} \varepsilon_{\alpha} \frac{\partial \psi^{\alpha}}{\partial u^{s}} \frac{\partial \psi^{\alpha}}{\partial u^{k}} \\
g^{i j}=\eta^{i s} \frac{\partial F^{j}}{\partial u^{s}}+\eta^{j s} \frac{\partial F^{i}}{\partial u^{s}}-\eta^{i s} \eta^{j k} \sum_{\alpha=1}^{L} \varepsilon_{\alpha} \frac{\partial \psi^{\alpha}}{\partial u^{s}} \frac{\partial \psi^{\alpha}}{\partial u^{k}} \\
b_{k}^{i j}=\eta^{i s} \frac{\partial^{2} F^{j}}{\partial u^{s} \partial u^{k}}-\eta^{i s} \eta^{j p} \sum_{\alpha=1}^{L} \varepsilon_{\alpha} \frac{\partial^{2} \psi^{\alpha}}{\partial u^{s} \partial u^{k}} \frac{\partial \psi^{\alpha}}{\partial u^{p}}
\end{gathered}
$$

При этом легко проверить, что для скобки Пуассона (3.1) выполнены все соотношения согласованности (2.3)-(2.6). 


\section{4. ИНТЕГРИРУЕМЫЕ УРАВНЕНИЯ ДЛЯ КАНОНИЧЕСКОЙ СОГЛАСОВАННОЙ ПАРЫ СКОБОК}

ТЕОРема 4.1. Нелокальная скобка (3.1) является скобкой Пуассона тогда и только тогда, когда выполнены следующие соотношения:

$$
\begin{aligned}
\frac{\partial^{2} Q_{1}}{\partial u^{i} \partial u^{s}} \eta^{s p} \frac{\partial^{2} Q_{2}}{\partial u^{p} \partial u^{j}} & =\frac{\partial^{2} Q_{2}}{\partial u^{i} \partial u^{s}} \eta^{s p} \frac{\partial^{2} Q_{1}}{\partial u^{p} \partial u^{j}}, \\
g_{\mathrm{can}}^{i s} \eta^{j r} \frac{\partial^{2} Q}{\partial u^{r} \partial u^{s}} & =g_{\text {can }}^{j s} \eta^{i r} \frac{\partial^{2} Q}{\partial u^{r} \partial u^{s}},
\end{aligned}
$$

$2 \partial e$

$$
g_{\mathrm{can}}^{i j}(u)=\eta^{i s} \frac{\partial F^{j}}{\partial u^{s}}+\eta^{j s} \frac{\partial F^{i}}{\partial u^{s}}-\eta^{i s} \eta^{j k} \sum_{\alpha=1}^{L} \varepsilon_{\alpha} \frac{\partial \psi^{\alpha}}{\partial u^{s}} \frac{\partial \psi^{\alpha}}{\partial u^{k}}
$$

- метрика скобки (3.1), функции $Q(u), Q_{1}(u)$ и $Q_{2}(u)$ - любъе из функций $F^{i}(u)$, $1 \leqslant i \leqslant N, u \psi^{\alpha}(u), \quad 1 \leqslant \alpha \leqslant L$.

Система (4.1) интегрируема методом обратной задачи рассеяния. Процедура интегрирования и пара Лакса со спектральным параметром для этой системы будут опубликованы в нашей последующей работе.

\section{5. ЛИУВИЛЛЕВЫ И СПЕЦИАЛЬНЫЕ ЛИУВИЛЛЕВЫ КООР ДИНАТЫ}

Локальные координаты $u=\left(u^{1}, \ldots, u^{N}\right)$ называются лиувиллевы.ми для произвольной скобки Пуассона $\{I, J\}$, если функции (поля) $u^{i}(x)$ являются плотностями интегралов в инволюции относительно этой скобки, т.е.

$$
\left\{U^{i}, U^{j}\right\}=0, \quad 1 \leqslant i, j \leqslant N
$$

где

$$
U^{i}=\int u^{i}(x) d x, \quad 1 \leqslant i \leqslant N
$$

В этом случае скобка Пуассона также называется лиувиллевой в этих координатах. Лиувиллевы координаты естественно возникают и играют существенную роль в процедуре Дубровина-Новикова усреднения гамильтоновых уравнений [3]. Физические координаты, получаемые усреднением плотностей участвуюших в процедуре Дубровина-Новикова $N$ инволютивных локальных интегралов изначальной гамильтоновой системы, всегда являются лиувиллевыми для соответствуюшей усредненной скобки. Это свойство и явилось мотивировкой определения лиувиллевых координат для локальных скобок Пуассона гидродинамического типа в работе [3]. Для общих нелокальных скобок Пуассона гидродинамического типа (1.8) лиувиллевы координаты введены в работе [15]. 
Нелокальная скобка Пуассона гидродинамического типа (1.8) является лиувиллевой в локальных координатах $u=\left(u^{1}, \ldots, u^{N}\right)$ тогда и только тогда, когда существуют матричная функция $\Phi^{i j}(u)$ и функции $\left(\varphi^{\alpha}\right)^{i}(u), 1 \leqslant \alpha \leqslant L$, такие, что скобка имеет следующий вид (см. [15], где именно это свойство взято за определение лиувиллевости скобки (1.8)):

$$
\begin{aligned}
\{I, J\}_{1}= & \int \frac{\delta I}{\delta u^{i}(x)}\left(\left[\Phi^{i j}(u)+\Phi^{j i}(u)-\sum_{\alpha=1}^{L} \varepsilon_{\alpha}\left(\varphi^{\alpha}\right)^{i}\left(\varphi^{\alpha}\right)^{j}\right] \frac{d}{d x}+\right. \\
& +\left[\frac{\partial \Phi^{i j}}{\partial u^{k}}-\sum_{\alpha=1}^{L} \varepsilon_{\alpha} \frac{\partial\left(\varphi^{\alpha}\right)^{i}}{\partial u^{k}}\left(\varphi^{\alpha}\right)^{j}\right] u_{x}^{k}+ \\
& \left.+\sum_{\alpha=1}^{L} \varepsilon_{\alpha} \frac{\partial\left(\varphi^{\alpha}\right)^{i}}{\partial u^{k}} u_{x}^{k}\left(\frac{d}{d x}\right)^{-1} \frac{\partial\left(\varphi^{\alpha}\right)^{j}}{\partial u^{s}} u_{x}^{s}\right) \frac{\delta J}{\delta u^{j}(x)} d x
\end{aligned}
$$

Из теоремы 3.1 следует

ТЕОРемА 5.1. Плоские координаты произвольной невырожденной локальной скобки Пуассона гидродинамического типа $\{I, J\}_{2}$ всегда являются лиувиллевыми для любой нелокальной скобки Пуассона $\{I, J\}_{1}(1.8)$, согласованной $c\{I, J\}_{2}$. Более того, при этом соответствующая лиувиллева функиия $\Phi^{i j}($ и) и функиии $\left(\varphi^{\alpha}\right)^{i}(u)$ всегда имеют специальный вид:

$$
\Phi^{i j}(u)=\eta^{i s} \frac{\partial F^{j}}{\partial u^{s}}, \quad\left(\varphi^{\alpha}\right)^{i}(u)=\eta^{i s} \frac{\partial \psi^{\alpha}}{\partial u^{s}}
$$

Локальные координаты $u=\left(u^{1}, \ldots, u^{N}\right)$ называются специальнылми лиувиллевым. координатами для произвольной скобки Пуассона $\{I, J\}$, если существует ненулевая постоянная симметричная матрица $\eta_{i j}$ такая, что функции (поля) $u^{i}(x), 1 \leqslant i \leqslant N$, и $\eta_{i j} u^{i}(x) u^{j}(x)$ являются плотностями интегралов в инволюции относительно этой скобки, т.е.

$$
\left\{U^{i}, U^{j}\right\}=0, \quad 1 \leqslant i, j \leqslant N+1
$$

где

$$
U^{i}=\int u^{i}(x) d x, \quad 1 \leqslant i \leqslant N ; \quad U^{N+1}=\int \eta_{i j} u^{i}(x) u^{j}(x) d x .
$$

В этом случае скобка Пуассона также называется специальной лиувиллевой в этих координатах. Специальные лиувиллевы координаты были введены в работах [16]. Наиболее важным является случай невырожденной матрицы $\eta_{i j}$, и именно этот случай мы и будем всегда иметь в виду.

ТЕОРема 5.2. Нелокальная скобка Пуассона вида (1.8) является специальной лиувиллевой в локальньх координатах $u=\left(u^{1}, \ldots, u^{N}\right)$ тогда и только тогда, когда она лиувиллева с лиувиллевой функиией $\Phi^{i j}(u)$ и функциями $\left(\varphi^{\alpha}\right)^{i}(u)$ специального вида такими, что

$$
\eta_{k s} \Phi^{s j}(u)=\frac{\partial F^{j}}{\partial u^{k}}, \quad \eta_{k s}\left(\varphi^{\alpha}\right)^{s}(u)=\frac{\partial \psi^{\alpha}}{\partial u^{k}} .
$$


Для невырожденной матрищы $\eta_{i j}$ получаем в этом случае в точности скобку $(3.1)$ из канонической согласованной пары.

Таким образом, задача о согласованных нелокальных скобках Пуассона гидродинамического типа эквивалентна задаче классификации специальных лиувиллевых координат для нелокальных скобок Пуассона гидродинамического типа.

ТЕОрема 5.3. Произвольная нелокальная скобка Пуассона гидродинамического типа (1.8) согласована с постоянной скобкой Пуассона (2.1) тогда и только тогда, когда функиии $u^{i}(x), 1 \leqslant i \leqslant N, u \eta_{i j} u^{i}(x) u^{j}(x), \eta^{i s} \eta_{s j}=\delta_{j}^{i}$, являются плотностями интегралов в инволюции относительно скобки Пуассона (1.8).

Отметим, что функции $u^{i}(x), 1 \leqslant i \leqslant N$, представляют собой плотности аннуляторов скобки (2.1), а $\eta_{i j} u^{i}(x) u^{j}(x) / 2$ - плотность импульса скобки (2.1).

ТЕОрема 5.4. Произвольная нелокальная скобка Пуассона гидродинамического типа (1.8) согласована с произвольной невырожденной локальной скобкой Пуассона (1.2) тогда и только тогда, когда $N$ аннуляторов и импульс скобки (1.2) являются интегралами в инволючии относительно скобки Пуассона (1.8).

\section{6. ИНТЕГРИРУЕМЫЕ БИГАМИЛЬТОНОВЫ ИЕРАРХИИ}

Рассмотрим пару согласованных гамильтоновых операторов гидродинамического типа $P_{1}^{i j}$ и $P_{2}^{i j}$, один из которых, скажем $P_{2}^{i j}$, локальный, а другой - произвольный нелокальный оператор вида оператора скобки (1.8). По-видимому, один из гамильтоновых операторов всегда можно привести к каноническому постоянному виду серией преобразований по решению, если он невырожден (аналог классической теоремы Дарбу в симплектической геометрии), так что рассматриваемьй случай является по сути общим. Если локальный гамильтонов оператор $P_{2}^{i j}$ невырожденный, то из теоремы 3.1 следует, что локальной заменой координат пара согласованных гамильтоновых операторов $P_{1}^{i j}$ и $P_{2}^{i j}$ приводится к следуюшему каноническому виду:

$$
\begin{gathered}
P_{2}^{i j}[u(x)]=\eta^{i j} \frac{d}{d x} \\
P_{1}^{i j}[u(x)]=\left(\eta^{i s} \frac{\partial F^{j}}{\partial u^{s}}+\eta^{j s} \frac{\partial F^{i}}{\partial u^{s}}-\eta^{i s} \eta^{j k} \sum_{\alpha=1}^{L} \varepsilon_{\alpha} \frac{\partial \psi^{\alpha}}{\partial u^{s}} \frac{\partial \psi^{\alpha}}{\partial u^{k}}\right) \frac{d}{d x}+ \\
+\left(\eta^{i s} \frac{\partial^{2} F^{j}}{\partial u^{s} \partial u^{k}}-\eta^{i s} \eta^{j p} \sum_{\alpha=1}^{L} \varepsilon_{\alpha} \frac{\partial^{2} \psi^{\alpha}}{\partial u^{s} \partial u^{k}} \frac{\partial \psi^{\alpha}}{\partial u^{p}}\right) u_{x}^{k}+ \\
+\eta^{i p} \eta^{j r} \sum_{\alpha=1}^{L} \varepsilon_{\alpha} \frac{\partial^{2} \psi^{\alpha}}{\partial u^{p} \partial u^{k}} u_{x}^{k}\left(\frac{d}{d x}\right)^{-1} \frac{\partial^{2} \psi^{\alpha}}{\partial u^{r} \partial u^{s}} u_{x}^{s}
\end{gathered}
$$

где $\eta^{i j}$ - произвольная невырожденная постоянная симметричная матрища $(1.6) ; F^{i}(u)$, $1 \leqslant i \leqslant N$, и $\psi^{\alpha}(u), 1 \leqslant \alpha \leqslant L,-$ гладкие функции, заданные в некоторой области локальных координат, такие, что оператор (6.2) является гамильтоновым, т.е. функции $F^{i}(u)$ и $\psi^{\alpha}(u)$ удовлетворяют интегрируемым уравнениям (4.1) (см. теорему 4.1). 
ЗАмЕчАниЕ. Очевидно, что всегда можно считать, что $\eta^{i j}=\varepsilon^{i} \delta^{i j}, \varepsilon^{i}=1$ при $i \leqslant p$, $\varepsilon^{i}=-1$ при $i>p$, где $p$-положительный индекс инерции метрики, $0 \leqslant p \leqslant N$, и, кроме того, необходимо классифишировать гамильтоновы операторы (6.2) относительно действия группы движений соответствуюшего $N$-мерного псевдоевклидова пространства $\mathbb{R}_{p}^{N}$, но для наших целей достаточно (и удобнее) пользоваться указанным выше представлением канонической согласованной пары ( "условно каноническим" представлением).

Рассмотрим оператор рекурсии, задаваемый каноническими согласованными гамильтоновыми операторами (6.1), (6.2),

$$
\begin{aligned}
R_{l}^{i}[u(x)]= & {\left[P_{1}[u(x)]\left(P_{2}[u(x)]\right)^{-1}\right]_{l}^{i}=} \\
= & \left(\left(\eta^{i s} \frac{\partial F^{j}}{\partial u^{s}}+\eta^{j s} \frac{\partial F^{i}}{\partial u^{s}}-\eta^{i s} \eta^{j k} \sum_{\alpha=1}^{L} \varepsilon_{\alpha} \frac{\partial \psi^{\alpha}}{\partial u^{s}} \frac{\partial \psi^{\alpha}}{\partial u^{k}}\right) \frac{d}{d x}+\right. \\
& +\left(\eta^{i s} \frac{\partial^{2} F^{j}}{\partial u^{s} \partial u^{k}}-\eta^{i s} \eta^{j p} \sum_{\alpha=1}^{L} \varepsilon_{\alpha} \frac{\partial^{2} \psi^{\alpha}}{\partial u^{s} \partial u^{k}} \frac{\partial \psi^{\alpha}}{\partial u^{p}}\right) u_{x}^{k}+ \\
& \left.+\eta^{i p} \eta^{j r} \sum_{\alpha=1}^{L} \varepsilon_{\alpha} \frac{\partial^{2} \psi^{\alpha}}{\partial u^{p} \partial u^{k}} u_{x}^{k}\left(\frac{d}{d x}\right)^{-1} \frac{\partial^{2} \psi^{\alpha}}{\partial u^{r} \partial u^{s}} u_{x}^{s}\right) \eta_{j l}\left(\frac{d}{d x}\right)^{-1}
\end{aligned}
$$

(об операторах рекурсии, задаваемых парой согласованных гамильтоновых операторов, см. [17]-[22]). Применим оператор рекурсии (6.3) к системе трансляций по $x$, т.е. к системе гидродинамического типа

$$
u_{t}^{i}=u_{x}^{i}
$$

которая, очевидно, является гамильтоновой с гамильтоновым оператором (6.1):

$$
u_{t}^{i}=u_{x}^{i} \equiv P_{2}^{i j} \frac{\delta H}{\delta u^{j}(x)}, \quad H=\frac{1}{2} \int \eta_{j l} u^{j}(x) u^{l}(x) d x .
$$

Любая система из иерархии

$$
u_{t_{n}}^{i}=\left(R^{n}\right)_{j}^{i} u_{x}^{j}, \quad n \in \mathbb{Z},
$$

является мультигамильтоновой интегрируемой системой. В частности, интегрируемой является любая система вида

$$
u_{t_{1}}^{i}=R_{j}^{i} u_{x}^{j},
$$

т.е. система гидродинамического типа

$$
\begin{aligned}
u_{t_{1}}^{i}= & \left(\left(\eta^{i s} \frac{\partial F^{j}}{\partial u^{s}}+\eta^{j s} \frac{\partial F^{i}}{\partial u^{s}}-\eta^{i s} \eta^{j k} \sum_{\alpha=1}^{L} \varepsilon_{\alpha} \frac{\partial \psi^{\alpha}}{\partial u^{s}} \frac{\partial \psi^{\alpha}}{\partial u^{k}}\right) \frac{d}{d x}+\right. \\
& +\left(\eta^{i s} \frac{\partial^{2} F^{j}}{\partial u^{s} \partial u^{k}}-\eta^{i s} \eta^{j p} \sum_{\alpha=1}^{L} \varepsilon_{\alpha} \frac{\partial^{2} \psi^{\alpha}}{\partial u^{s} \partial u^{k}} \frac{\partial \psi^{\alpha}}{\partial u^{p}}\right) u_{x}^{k}+
\end{aligned}
$$




$$
\begin{aligned}
& \left.+\eta^{i p} \eta^{j r} \sum_{\alpha=1}^{L} \varepsilon_{\alpha} \frac{\partial^{2} \psi^{\alpha}}{\partial u^{p} \partial u^{k}} u_{x}^{k}\left(\frac{d}{d x}\right)^{-1} \frac{\partial^{2} \psi^{\alpha}}{\partial u^{r} \partial u^{s}} u_{x}^{s}\right) \eta_{j l} u^{l} \equiv \\
\equiv & \left(\eta^{i s} \frac{\partial F^{j}}{\partial u^{s}} \eta_{j k}+\frac{\partial F^{i}}{\partial u^{k}}+\eta^{i s} \frac{\partial^{2} F^{j}}{\partial u^{s} \partial u^{k}} \eta_{j r} u^{r}-\right. \\
& \left.-\eta^{i s} \sum_{\alpha=1}^{L} \varepsilon_{\alpha} \frac{\partial \psi^{\alpha}}{\partial u^{s}} \frac{\partial \psi^{\alpha}}{\partial u^{k}}-\eta^{i s} \sum_{\alpha=1}^{L} \varepsilon_{\alpha} \frac{\partial^{2} \psi^{\alpha}}{\partial u^{s} \partial u^{k}} \psi^{\alpha}\right) u_{x}^{k} \equiv \\
\equiv & \left(F^{i}(u)+\eta^{i s} \eta_{j r} \frac{\partial F^{j}}{\partial u^{s}} u^{r}-\eta^{i s} \sum_{\alpha=1}^{L} \varepsilon_{\alpha} \frac{\partial \psi^{\alpha}}{\partial u^{s}} \psi^{\alpha}\right)_{x},
\end{aligned}
$$

где $F^{i}(u), \quad 1 \leqslant i \leqslant N$, и $\psi^{\alpha}(u), \quad 1 \leqslant \alpha \leqslant L,-$ произвольное решение интегрируемой системы (4.1). Эта система гидродинамического типа бигамильтонова с парой канонических согласованных гамильтоновых операторов (6.1), (6.2):

$$
\begin{aligned}
u_{t_{1}}^{i}= & \left(\left(\eta^{i s} \frac{\partial F^{j}}{\partial u^{s}}+\eta^{j s} \frac{\partial F^{i}}{\partial u^{s}}-\eta^{i s} \eta^{j k} \sum_{\alpha=1}^{L} \varepsilon_{\alpha} \frac{\partial \psi^{\alpha}}{\partial u^{s}} \frac{\partial \psi^{\alpha}}{\partial u^{k}}\right) \frac{d}{d x}+\right. \\
& +\left(\eta^{i s} \frac{\partial^{2} F^{j}}{\partial u^{s} \partial u^{k}}-\eta^{i s} \eta^{j p} \sum_{\alpha=1}^{L} \varepsilon_{\alpha} \frac{\partial^{2} \psi^{\alpha}}{\partial u^{s} \partial u^{k}} \frac{\partial \psi^{\alpha}}{\partial u^{p}}\right) u_{x}^{k}+ \\
& \left.+\eta^{i p} \eta^{j r} \sum_{\alpha=1}^{L} \varepsilon_{\alpha} \frac{\partial^{2} \psi^{\alpha}}{\partial u^{p} \partial u^{k}} u_{x}^{k}\left(\frac{d}{d x}\right)^{-1} \frac{\partial^{2} \psi^{\alpha}}{\partial u^{r} \partial u^{s}} u_{x}^{s}\right) \frac{\delta H_{1}}{\delta u^{j}(x)}, \\
H_{1}=\frac{1}{2} \int \eta_{j l} u^{j}(x) u^{l}(x) d x ; & \left.H_{t_{1}}^{i}=\eta^{i j} \frac{d}{d x} \frac{\delta H_{2}}{\delta u^{j}(x)}, \quad H_{2}=\int \eta_{j k}(u(x)) u^{j}(x)-\frac{1}{2} \sum_{\alpha=1}^{L} \varepsilon_{\alpha}\left(\psi^{\alpha}(u)\right)^{2}\right) d x .
\end{aligned}
$$

Благодарности. Работа выполнена при финансовой поддержке Фонда Александра фон Гумбольдта (Германия), а также Российского фонда фундаментальных исследований (грант № 02-01-00803) и INTAS (грант № 99-1782).

\section{Список литературы}

[1] О. И. Мохов. Функц. анализ и его прилож. 2001. Т. 35. № 2. С. 24-36; math.DG/0005051.

[2] О.И. Мохов. УМН. 2000. Т. 55. № 4. С. 217-218.

[3] Б. А. Дубровин, С. П. Новиков. ДАН СССР. 1983. Т. 270. № 4. С. 781-785.

[4] O.I. Mokhov. Compatible Dubrovin-Novikov Hamiltonian operators, Lie derivative and integrable systems of hydrodynamic type. Proc. of the International Conference "Nonlinear Evolution Equations and Dynamical Systems" (Cambridge, July 24-30, 2001). (to appear); math.DG/0201281.

[5] О.И. Мохов. УМН. 2002. Т. 57. № 1. С. 157-158.

[6] О.И. Мохов, Е. В. Ферапонтов. УМН. 1990. Т. 45. № 3. С. 191-192.

[7] O. I. Mokhov. Liouville canonical form for the compatible nonlocal Poisson brackets of hydrodynamic type, and integrable hierarchies. math.DG/0201223.

[8] Б. А. Дубровин, С. П. Новиков. УМН. 1989. Т. 44. № 6. С. $29-98$. 
[9] С. П. Царёв. ДАН СССР. 1985. Т. 282. № 3. С. 534-537.

[10] С. П. Царёв. Изв. АН СССР. Сер. матем. 1990. Т. 54. № 5. С. 1048-1068.

[11] E. В. Ферапонтов. Функц. анализ и его прилож. 1991. Т. 25. № 3. С. 37-49.

[12] O. I. Mokhov. Phys. Lett. A. 1992. V. 166. № 3-4. P. 215-216.

[13] О. И. Мохов, Е. В. Ферапонтов. Функц. анализ и его прилож. 1994. Т. 28. № 2. С. 60-63.

[14] F. Magri. J. Math. Phys. 1978. V. 19. № 5. P. 1156-1162.

[15] A. Ya. Maltsev, S. P. Novikov. Physica D. 2001. V. 156. № 1-2. P. 53-80; nlin.SI/0006030.

[16] О.И. Мохов. Тр. МИРАН. 1999. Т. 225. С. 284-300; O. I. Mokhov. Rep. Math. Phys. 1999. V. 43. № 1-2. Р. 247-256.

[17] И. М. Гельфанд, И. Я. Дорфман. Функц. анализ и его прилож. 1979. Т. 13. № 4. С. 13-30.

[18] B. Fuchssteiner. Nonlinear Anal. Theor. Meth. Appl. 1979. V. 3. P. 849-862.

[19] A.S. Fokas, B. Fuchssteiner. Lett. Nuovo Cimento. 1980. V. 28. № 8. P. 299-303.

[20] П. Олвер. Приложения групп Ли к дифференциальным уравнениям. М.: Мир, 1989.

[21] I. Dorfman. Dirac Structures and Integrability of Nonlinear Evolution Equations. Chichester: John Wiley \& Sons, 1993.

[22] О.И. Мохов. УМН. 1998. Т. 53. № 3. С. 85-192. 